\title{
PIM Kinases Promote Survival and Immune Escape in Primary Mediastinal Large B-Cell Lymphoma through Modulation of JAK-STAT and NF- $\kappa$ B Activity
}

\author{
Maciej Szydłowski, ${ }^{*}$ Sonia Dębek, ${ }^{*}$ Monika Prochorec-Sobieszek, ${ }^{\dagger}$ Małgorzata Szołkowska, ${ }^{\ddagger}$ Andrea M. Tomirotti, \\ Przemysław Juszczyński, ${ }^{*}$ and Anna Szumera-Ciećkiewicz
}

From the Departments of Experimental Hematology* and Diagnostic Hematology, ${ }^{\dagger}$ Institute of Hematology and Transfusion Medicine, Warsaw, Poland; the Department of Pathology, ${ }^{\ddagger}$ The National Tuberculosis and Lung Diseases Research Institute, Warsaw, Poland; and the Department of Experimental and Translational Oncology, ${ }^{\S}$ Menarini Ricerche, Pomezia, Italy

\author{
Accepted for publication \\ December 1, 2020. \\ Address correspondence to \\ Maciej Szydłowski, Ph.D., \\ Department of Experimental \\ Hematology, Institute of Hema- \\ tology and Transfusion Medi- \\ cine, Indiry Gandhi 14, Warsaw \\ 02-776, Poland. E-mail: \\ mszydlowski@ihit.waw.pl.
}

\begin{abstract}
Primary mediastinal large B-cell lymphoma (PMBL) cells depend on the constitutive activity of NF- $\kappa \mathrm{B}$ and STAT transcription factors, which drive expression of multiple molecules essential for their survival. In a molecularly related B-cell malignant tumor (classic Hodgkin lymphoma), tumor Reed-Sternberg cells overexpress oncogenic (proviral integration site for Moloney murine leukemia virus (PIM) 1, 2, and 3 kinases in a NF-KB- and STAT-dependent manner and PIMs enhance survival and expression of immunomodulatory molecules. Given the multiple overlapping characteristics of Reed-Sternberg and PMBL cells, we hypothesized that PIM kinases may be overexpressed in PMBL and involved in PMBL pathogenesis. The expression of PIM kinases in PMBL diagnostic biopsy specimens was assessed and their role in survival and immune escape of the tumor cells was determined. PIMs were abundantly expressed in primary tumors and PMBL cell lines. Inhibition of PIM kinases was toxic to PMBL cells, attenuated protein translation, and down-regulated NF- $\kappa \mathrm{B}-$ and STAT-dependent transcription of prosurvival factors $B C L 2 A 1, B C L 2 L 1$, and FCER2. Furthermore, PIM inhibition decreased expression of molecules engaged in shaping the immunosuppressive microenvironment, including programmed death ligand $1 / 2$ and chemokine (C-C motif) ligand 17. Taken together, our data indicate that PIMs support PMBL cell survival and immune escape and identify PIMs as promising therapeutic targets for PMBL. (Am J Pathol 2021, 191: 567-574; https://doi.org/10.1016/j.ajpath.2020.12.001)
\end{abstract}

Primary mediastinal large B-cell lymphoma (PMBL) is an aggressive B-cell lymphoma, accounting for approximately $2 \%$ to $4 \%$ of all mature B-cell neoplasms, affecting predominantly young girls, and manifesting with a large mediastinal mass that arises from B cells of a thymic origin. ${ }^{1}$ Although cure rates with standard immunochemotherapy, such as R-CHOP (rituximab, cyclophosphamide, doxorubicin, vincristine, and prednisone) or DA-EPOCH-R (etoposide, doxorubicin, cyclophosphamide, vincristine, prednisone, and rituximab), reach $90 \%$, treatment of patients with relapsed/refractory PMBL remains largely ineffective. In those patients, new therapeutic approaches are required to improve prognosis.
Historically, PMBL was designated as a subtype of diffuse large B-cell lymphoma (DLBCL). However, subsequent molecular studies revealed multiple differences with DLBCL and highlighted common features shared by PMBL and nodular sclerosis classic Hodgkin lymphoma (cHL), including transcriptional profile, genomic aberrations

Supported by National Science Centre Poland grant 2016/22/M/NZ5/ 00668 and Project Infrastructure grant POIG.02.03.00-14-111/13 from the Operational Programme Innovative Economy 2007 to 2013, Priority II, R\&D Infrastructure, Measure 2.3 (investments connected with the development of the information technology infrastructure of science).

P.J. and A.S.-C. contributed equally to this work.

Disclosures: A.M.T. is a Menarini Ricerche employee. 
leading to overexpression of programmed death ligand (PDL) 1 and -2 , microenvironmental interactions, or addiction to NF- $\kappa \mathrm{B}$ and JAK-STAT signaling pathways. ${ }^{2-4} \mathrm{NF}-\kappa \mathrm{B}$ and STAT transcription factors drive expression of the proviral integration site for Moloney murine leukemia virus (PIM) 1, 2, and 3 kinases in cHL Reed-Sternberg (RS) cells and PIMs boost the expression of antiapoptotic proteins, cytokines, chemokines, and immunomodulatory molecules, including PD-L1 and PD-L2, thus fostering RS cell survival and immune escape. ${ }^{5}$ Given the common molecular features of RS and PMBL cells, we hypothesized that PIMs may be overexpressed in PMBL and thus might contribute to the pathogenesis of the disease. PIM1, -2 , and -3 are abundantly expressed in primary tumors and PMBL cell lines. Inhibition of PIMs in PMBL cells decreases proliferation, induces apoptosis, and down-regulates expression of antiapoptotic factors and molecules engaged in the formation of immunosuppressive niche in PMBL.

\section{Materials and Methods}

\section{Cell Lines, Culture Conditions, and Chemicals}

Human PMBL cells Karpas1106P and U2940 and L-428 cHL cells were maintained in RPMI 1640 medium supplemented with $20 \%$ fetal bovine serum (Lonza Group AG, Basel, Switzerland). SUP-HD1 cHL cells were grown in McCoy's 5A medium supplemented with $20 \%$ fetal bovine serum (Lonza Group AG). B-cell acute lymphoblastic leukemia SEM cell line was cultured in Iscove's modified Dulbecco's medium supplemented with $10 \%$ fetal bovine serum (Lonza Group AG). The dual pan-PIM- FMS-like tyrosine kinase 3 (FLT3) inhibitor MEN1703 was provided by Menarini Ricerche (Pomezia, Italy), and the pan-PIM inhibitor PIM447 was purchased from Selleckchem (Houston, TX). All chemicals were dissolved in dimethyl sulfoxide (DMSO) (Sigma-Aldrich, St. Louis, MO). In vehicle-control experiments, final DMSO concentrations was $0.5 \%$ (control for a $5-\mu \mathrm{mol} / \mathrm{L}$ dose).

\section{Immunoblotting}

Protein lysates were resolved by SDS-PAGE, transferred to polyvinylidene difluoride membranes (Millipore, Burlington, MA), and immunoblotted with primary and appropriate horseradish peroxidase-labeled secondary antibodies. The following primary antibodies were used: anti-PIM1 (catalog number 54523; Cell Signaling Technology, Danvers, MA), anti-PIM2 (catalog number 4730; Cell Signaling Technology), anti-PIM3 (catalog number 4165; Cell Signaling Technology), anti-ph-STAT5-T694 (catalog number 9359; Cell Signaling Technology), anti-STAT5 (catalog number 9363; Cell Signaling Technology), anti-ph-STAT3-T705 (catalog number 9145; Cell Signaling Technology), antiSTAT3 (catalog number 21045; Signalway Antibody LLC, College Park, MD), anti-glyceraldehyde-3-phosphate dehydrogenase (GAPDH) (catalog number MAB374; Millipore), anti-ph-S6-S235/6 (catalog number 4857; Cell Signaling Technology), anti-S6 (catalog number 2217; Cell Signaling Technology), anti-ph-4EBP1-S65 (catalog number 9456; Cell Signaling Technology), anti-4EBP1 (catalog number 9644; Cell Signaling Technology), anti-ph-RelAS276 (catalog number 11011-1; SAB Biotherapeutics), antiRelA (catalog number SC-8008; Santa Cruz Biotechnology, Dallas, TX), and anti-FLT3 (catalog number 3462; Cell Signaling Technology). Chemiluminescent signals were detected using an ECL reagent (PerkinElmer, Waltham, MA) and the G:Box image acquisition system (Syngene International Ltd., Bangalore, India). To reprobe blots with another antibody, membranes were incubated in the stripping buffer for 30 minutes at $50^{\circ} \mathrm{C}$ followed by an incubation with another primary antibody. Band intensities were quantified by densitometry using ImageJ software version 1.52a $(\mathrm{NIH}$, Bethesda, MD; http://imagej.nih.gov/ij).

\section{Immunohistochemistry}

A retrospective group of 15 patients diagnosed according to the 2017 World Health Organization classification ${ }^{6}$ with primary mediastinal large B-cell lymphoma were studied. All patients provided consent for diagnostic workup of their diagnostic tissue specimens. Per local and institutional bioethical policies, research use of archival, diagnostic tissue specimens anonymously does not require special institutional review board approval and consent beyond the consent to perform a diagnostic workup. Immunohistochemistry on formalin-fixed, paraffin-embedded sections was performed using an automated stainer (Dako Denmark A/S, Glostrup, Denmark) and the following indicated antibodies: anti-PIM1 (ST0513) (1:100, pH 9.0; catalog number NBP2-67528; Novus Biologicals, Littleton, CO), anti-PIM2 (D1D2) (1:100, pH 9.0; Cell Signaling Technology), and anti-PIM3 (D17C9) (1:50, pH 9.0; Cell Signaling Technology). The EnVision Detection System (Dako Denmark $\mathrm{A} / \mathrm{S}$ ) was used for signal detection. Human prostate adenocarcinoma, testis, and hepatocellular carcinoma biopsy specimens were used as positive staining controls for PIM1, PIM2 and PIM3, respectively. Negative (isotype) control stainings were performed using a ready-to-use FLEX Negative Mouse Control (a cocktail of mouse IgG1, IgG2a, IgG2b, IgG3, and IgM; code number IR750; Dako Denmark A/S). Staining intensities in PMBL sections were scored using a semiquantitative method $(0$, no staining; 1 , weak, 2, intermediate, and 3, strong staining). Samples were considered positive for PIM1, PIM2, and PIM3 only if $\geq 10 \%$ of the lymphoma cells exhibited strong and/or intermediate reactions or when $\geq 20 \%$ were weakly positive. Analyses were performed independently by two hematopathologists (M.P.S. and A.S.C.) in a blinded fashion. All microphotographs were taken by a microscope DP72 Olympus BX63 camera (Olympus, Tokyo, Japan). 
PIMs Support Oncogenic Program in PMBL

\section{Real-Time Quantitative PCR}

RNA was extracted using a GeneMATRIX Universal RNA purification kit (EURx, Gdańsk, Poland) and reverse transcribed using a Superscript III Reverse Transcriptase (Invitrogen, Carlsbad, CA), according to the manufacturer's instructions. Gene expression levels were measured with the CFX Realtime System (Bio-Rad Laboratories, Hercules, CA) using the SYBR Green PCR Master Mix (Life Technologies, Carlsbad, CA) and the gene-specific primers (Table 1). Obtained $C_{T}$ values for target genes and housekeeping control (RPL29) were used to calculate relative transcript abundance using the $2^{-\Delta \Delta \mathrm{CT}}$ method. ${ }^{5}$ Experiments were conducted in triplicate, and differences in gene expression were compared with the Gosset's two-sided $t$-test, using GraphPad QuickCalcs/Prism software version 6 (GraphPad Software, San Diego, CA).

\section{Proliferation and Apoptosis Assays}

Proliferation was assessed using the CellTiter96 AQueous nonradioactive cell proliferation assay (Promega, Madison, WI). IC $_{50}$ values were calculated using GraphPad Prism version 6 software. All experiments were conducted in triplicate. For apoptosis, cells were washed in phosphatebuffered saline, suspended in $1 \times$ AnnexinV binding buffer (Becton, Dickinson and Co., Franklin Lakes, NJ), stained with AnnexinV-fluorescein isothiocyanate and propidium iodide (Becton, Dickinson and Co.), and analyzed using flow cytometry.

\section{NF- $\kappa B$ DNA Binding Assay}

DNA-binding activities of NF- $\kappa \mathrm{B}$ complexes were assessed using a colorimetric NF- $\kappa \mathrm{B}$ binding assay (TransAM NF-kB kit, catalog number 43296; Active Mofif Inc., Carlsbad, CA). A total of $20 \mu \mathrm{g}$ of protein extracts was added to the microwells. Signals were developed according to manufacturer's instructions and quantified using Multiskan GO plate reader (Thermo Fisher Scientific, Waltham, MA). Samples were measured in duplicates.

\section{Flow Cytometry}

PD-L1 and PD-L2 expression was assessed by flow cytometry as described previously, ${ }^{5}$ using phosphatidylethanolamineconjugated antibodies (BioLegend, San Diego, CA). Cells were analyzed using a Cytoflex flow cytometer (Beckman Coulter, Brea, CA).

\section{Statistical Analysis}

Differences among continuous variables were compared by the Gosset's two-sided $t$-test, using GraphPad QuickCalcs/Prism version 6 software (GraphPad Software).
Table 1 Primer Used in Real-Time Quantitative PCR Analysis

\begin{tabular}{ll}
\hline Primer & Sequence \\
\hline RPL29-F & $5^{\prime}$-CAGCTCAGGCTCCCAAAC-3' \\
RPL29-R & $5^{\prime}$-GCACCAGTCCTTCTGTCCTC-3' \\
NFKBIA-F & $5^{\prime}$-CCAACTACAATGGCCACACGT-3' \\
NFKBIA-R & $5^{\prime}$-TCCGGCCATTACAGGGCT-3' \\
TNF-F & $5^{\prime}$-AGGACGAACATCCAACCTTCCCAA-3' \\
TNF-R & $5^{\prime}$-TTTGAGCCAGAAGAGGTTGAGGGT-3' \\
CD40-F & $5^{\prime}$-AACAGGCAGGCACAAACAAGACTG-3' \\
CD40-R & $5^{\prime}$-TGGCAAACAGGATCCCGAAGATGA-3' \\
BCL2A1-F & $5^{\prime}$-CAGAAGATGACAGACTGTGAA-3' \\
BCL2A1-R & $5^{\prime}$-TCCAAGCATGACTTCAGATTC-3' \\
BCL2L1-F & $5^{\prime}$-GGATTTGAATCTCTTTCTCTCC-3' \\
BCL2L1-R & $5^{\prime}$-TCAACCACCAGCTCCCGGT-3' \\
CCL5-F & $5^{\prime}$-ACCATGAAGGTCTCCGCGG-3' \\
CCL5-R & $5^{\prime}$-GTGACAAAGACGACTGCTGG-3' \\
FCER2-F & $5^{\prime}$-AATCTCAGGACTTGGAGCTG-3' \\
FCER2-R & $5^{\prime}$-CCGCTGGACACCTGCAAC-3' \\
CCL17-F & $5^{\prime}$-CATCCACGCAGCTCGAGG-3' \\
CCL17-R & $5^{\prime}$-TCTTCACTCTCTTGTTGTTGG-3' \\
CD274-F & $5^{\prime}$-CATACAACAAAATCAACCAAAGA-3' \\
CD274-R & $5^{\prime}$-TGGTCTTACCACTCAGGACT-3' \\
\hline
\end{tabular}

$F$, forward; $R$, reverse.

\section{Results}

PIMs Are Ubiquitously Expressed in PMBL Cells

To determine the role of PIMs in PMBL, PIM-1/2/3 protein abundance in primary tumors and in two PMBL cell lines was first assessed. Twelve of 15 PMBL biopsy specimens exhibited expression of PIM1 (80\%) [4 biopsies (26\%) expressed PIM2 and 12 biopsies (80\%) expressed PIM3] (Figure 1A, Supplemental Table S1). Staining patterns of PIMs in PMBL cells were similar to those observed previously in RS and DLBCL cells. ${ }^{5,7}$ PIM1 and PIM2 exhibited nuclear and cytoplasmatic staining, whereas PIM3 appeared only in the cytosol. All investigated PMBL cases were positive for the expression of at least one PIM kinase isoform. PMBL cell lines Karpas1106P and U2940 expressed all three PIM kinases and exhibited higher PIM3 levels compared with two RS-derived cell lines, L-428 and SUP-HD1 (Figure 1B).

Expression of PIM kinases in RS cells is regulated by the

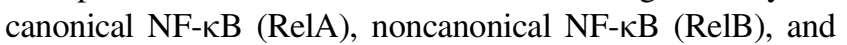
STAT transcription factors. ${ }^{5}$ We hypothesized that the aberrant activity of these factors may induce PIM expression in PMBL. Therefore, the activity of STAT3, STAT5, RelA, and RelB in PMBL cells was assessed. Similar to RS cells (L428 and SUP-HD1), Karpas1106P and U2940 cells expressed phosphorylated (T705) STAT3 but were phosphorSTAT5 negative (Figure 1C). RelA and RelB DNA-binding activities were comparable between PMBL and RS cells, with the highest levels of RelA and RelB activity observed in Karpas1106P PMBL cells (Figure 1D). Taken together, these data indicate that PIM kinases are abundantly expressed in 


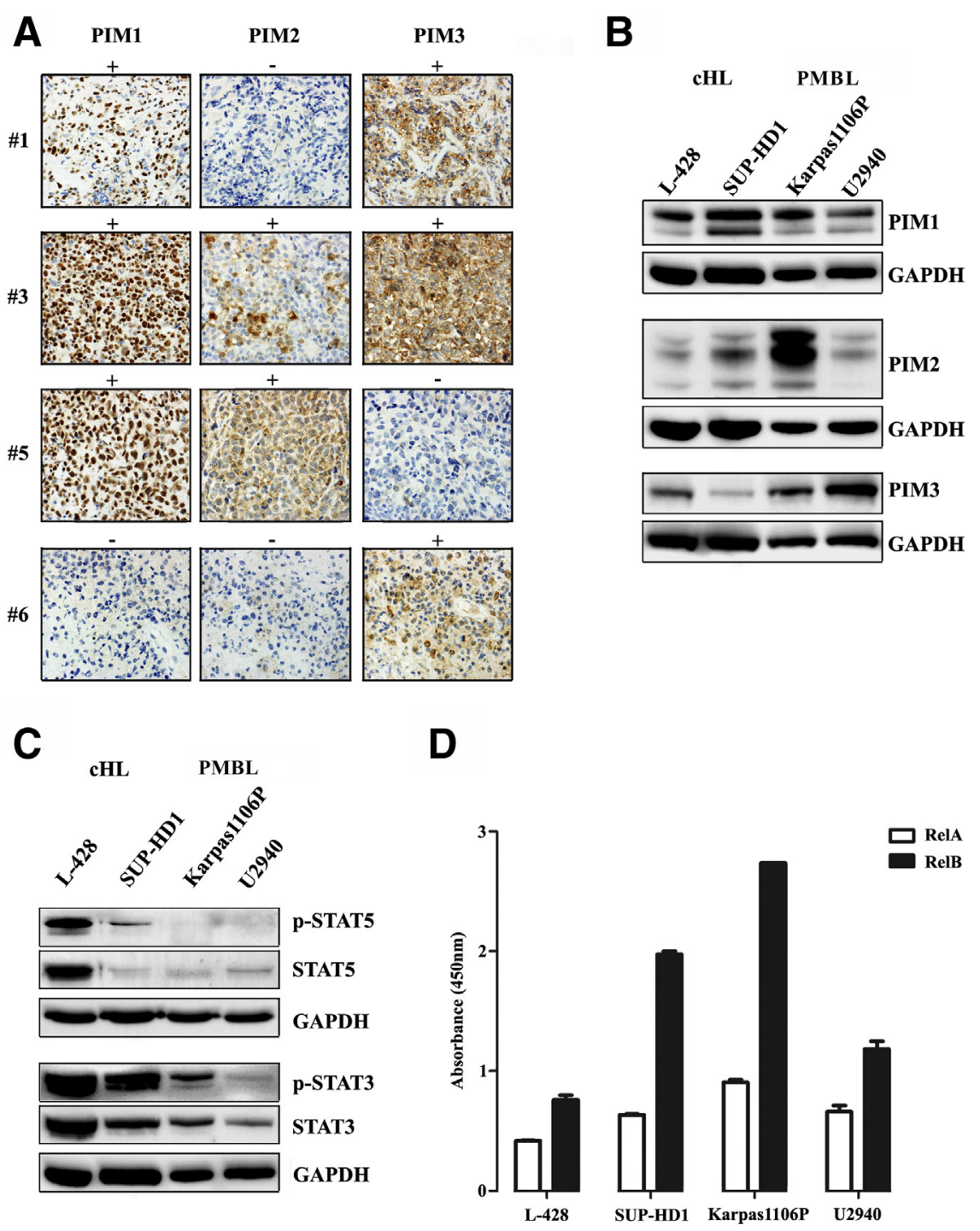

Figure 1 Primary mediastinal large B-cell lymphoma (PMBL) cells express proviral integration site for Moloney murine leukemia virus (PIM) 1, 2, and 3 kinases. A: Immunohistochemical analysis of PIM expression in PMBL samples. Shown are representative PMBL cases, double positive for PIM1 and PIM3 (patient 1), triple positive for PIM1, PIM2, and PIM3 (patient 3), double positive for PIM1 and PIM2 (patient 5), and positive for PIM3 (patient 6). B: Western blot analysis of PIM1, PIM2, and PIM3 expression in PMBL (Karpas1106P and U2940) and classical Hodgkin lymphoma ( $\mathrm{CHL}$ )-derived Reed-Sternberg cell lines (L-428 and SUP-HD1). Glyceraldehyde-3phosphate dehydrogenase (GAPDH) served as a loading control. C: Western blot analysis of STAT5/ phospho-STAT5 (p-STAT5), STAT3/phospho-STAT3 (p-STAT3) expression in PMBL and cHL cell lines. GAPDH served as a loading control. D: DNAbinding activity of the RelA- and RelB-containing $\mathrm{NF}-\kappa \mathrm{B}$ complexes. PMBL and $\mathrm{cHL}$ cells were lysed and subjected to TransAM DNA-binding assay using specific antibodies. Samples were measured in duplicate. Data are expressed as means \pm SD (D). $n=2$ independent experiments (D). Original magnification, $\times 400($ A) .
PMBL cells and that their expression might be related to the activity of NF- $\mathrm{BB}$ and STAT transcription factors.

\section{PIM Kinase Inhibition Decreases Protein Translation,} Attenuates NFKB-RelA and STAT3 Activity, and Induces Cell Apoptosis in PMBL Cells

PIM kinases support tumor cell viability and proliferation in certain B-cell lymphomas, including cHL. ${ }^{5,71}$ To address the role for PIM kinases in PMBL cells, proliferation and apoptosis of Karpas1106P and U2940 cells incubated with a dual pan-PIM/FLT3 inhibitor (MEN1703) were assessed. Because PMBL cells do not express FLT3, MEN1703 activity in this malignant tumor cannot be related to FLT3 inhibition (Figure 2A). MEN1703 markedly decreased proliferation of PMBL cells in a dose-dependent manner, with an $\mathrm{IC}_{50}$ of $0.97 \mu \mathrm{mol} / \mathrm{L}$ in Karpas $1106 \mathrm{P}$ and $1.75 \mu \mathrm{mol} /$ L in U2940 cells (Figure 2B). To determine whether PIM inhibition affects PMBL cell survival, Karpas1106P and
U2940 cells were cultured with MEN1703 (1.5 to $5 \mu \mathrm{mol} / \mathrm{L}$ ) or vehicle alone (DMSO) for 96 hours and evaluated by AnnexinV/propidium iodide staining. MEN1703 increased the fraction of apoptotic cells from $7.8 \%$ to $53 \%$ in Karpas $1106 \mathrm{P}$ cells and from $42.09 \%$ to $73.92 \%$ in U2940 cells (Figure 2C). Effects of PIM kinase inhibition on PMBL cell proliferation and apoptosis were confirmed using an additional, structurally unrelated, selective pan-PIM inhibitor (PIM447) (Supplemental Figure S1, A and B).

The cytotoxic activity of PIM kinase blockade in PMBL cells encouraged us to investigate its underlying molecular mechanisms. Given previously characterized functions of PIM kinases, cap-dependent protein translation (phosphorylation levels of 4EBP1 and the ribosomal protein S6) and the activity of STAT3 and NF- $\kappa \mathrm{B}-$ RelA transcription factors were evaluated. ${ }^{5,12,13}$ After incubation with either MEN1703 or PIM447, 4EBP1 and S6 phosphorylations in Karpas11106P and U2940 decreased in the dose-dependent manner (Figure 2D and Supplemental Figure S1C). PIM inhibition also 
A

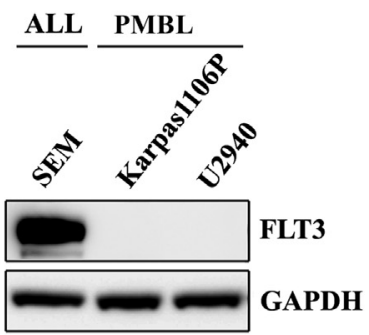

B

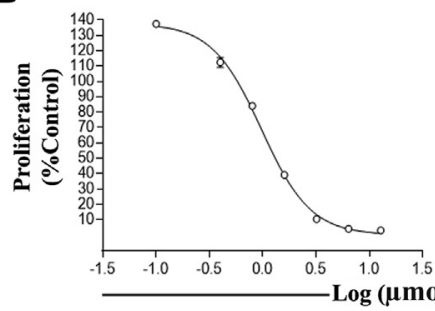

Ic50:
Karpas1106P

$0.9728 \mu \mathrm{mol} / \mathrm{L}$
U2940

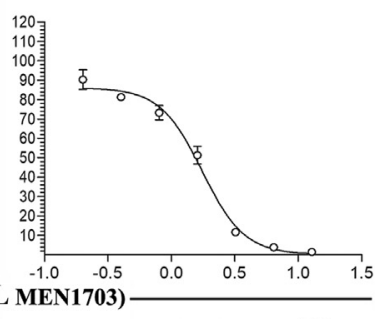

$1.749 \mu \mathrm{mol} / \mathrm{L}$

C

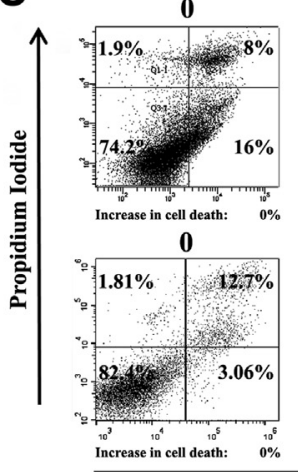

MEN1703 $(\mu \mathrm{mol} / \mathrm{L})$
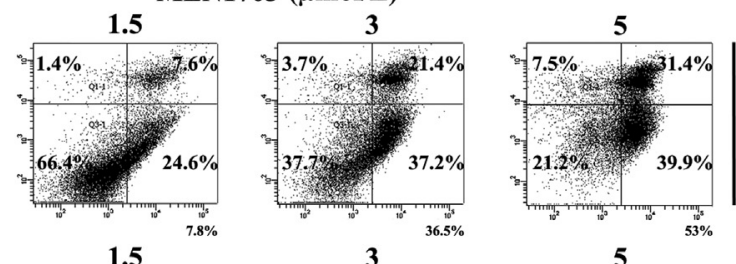

Karpas1106P
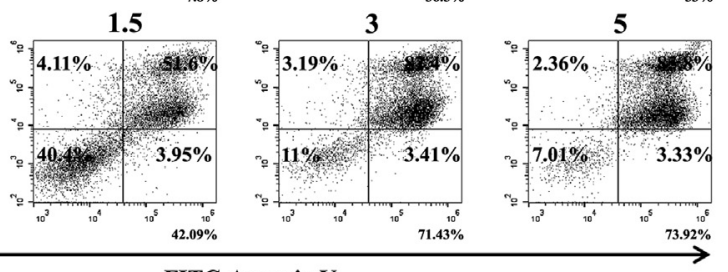

U2940

D

Karpas1106P

U2940
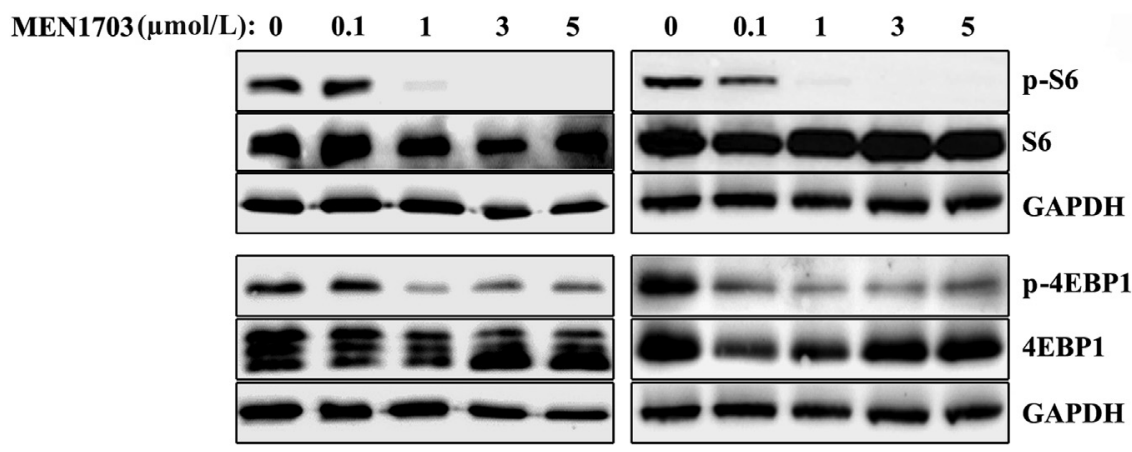

p-4EBP1
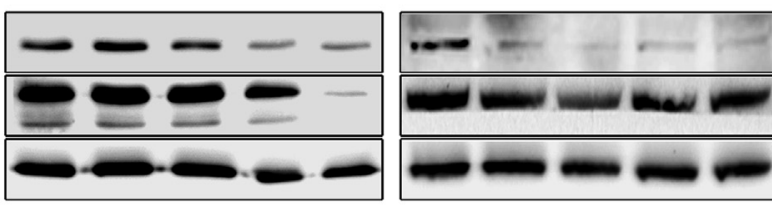

4EBP1

GAPDH
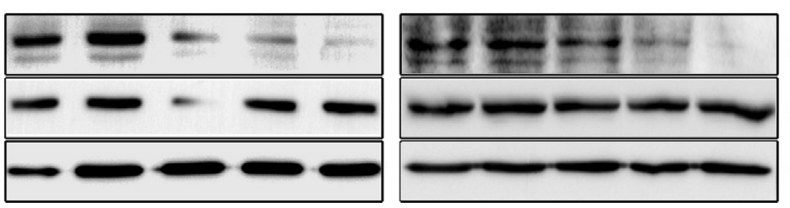

p-RelA

RelA

GAPDH

Figure 2 Proviral integration site for Moloney murine leukemia virus (PIM) inhibition increases primary mediastinal large B-cell lymphoma (PMBL) cell death and attenuates protein translation and NF- $\kappa B$ and STAT activity. A: FMS-like tyrosine kinase 3 (FLT3) protein expression in PMBL cells. Western blot analysis of the FLT3 protein in acute lymphoblastic leukemia (ALL) SEM cell line (used as a positive control) and the Karpas1106P and U2940 PMBL cells. Glyceraldehyde-3phosphate dehydrogenase (GAPDH) served as a loading control. B: IC $_{50}$ dose-response curve for MEN1703 in PMBL-derived Karpas1106P and U2940 cells. Cellular proliferation was determined by the CellTiter96 AQueous nonradioactive cell proliferation assay after 72 hours of incubation. C: Cellular apoptosis in MEN1703-treated Karpas1106P and U2940 cells. After 96 hours of incubation with 1.5 to $5 \mu \mathrm{mol} / \mathrm{L}$ of MEN1703, apoptosis was measured by AnnexinV/propidium iodide staining followed by fluorescence-activated cell sorting analysis. D: Immunoblot analyses of 4EBP1 (S65), S6(S235/236), RelA (S276), and STAT3 (T705) phosphorylations (p-) after MEN1703 incuabation. Cells were treated for 6 hours with dimethyl sulfoxide or with increasing doses ( 0.1 to $5 \mu$ mol/L) of MEN1703, harvested, and lysed. Glyceraldehyde-3-phosphate dehydrogenase (GAPDH) served as a loading control. Data are expressed as means \pm SD (B). $n=3$ independent experiments (B-D). FITC, fluorescein isothiocyanate. 

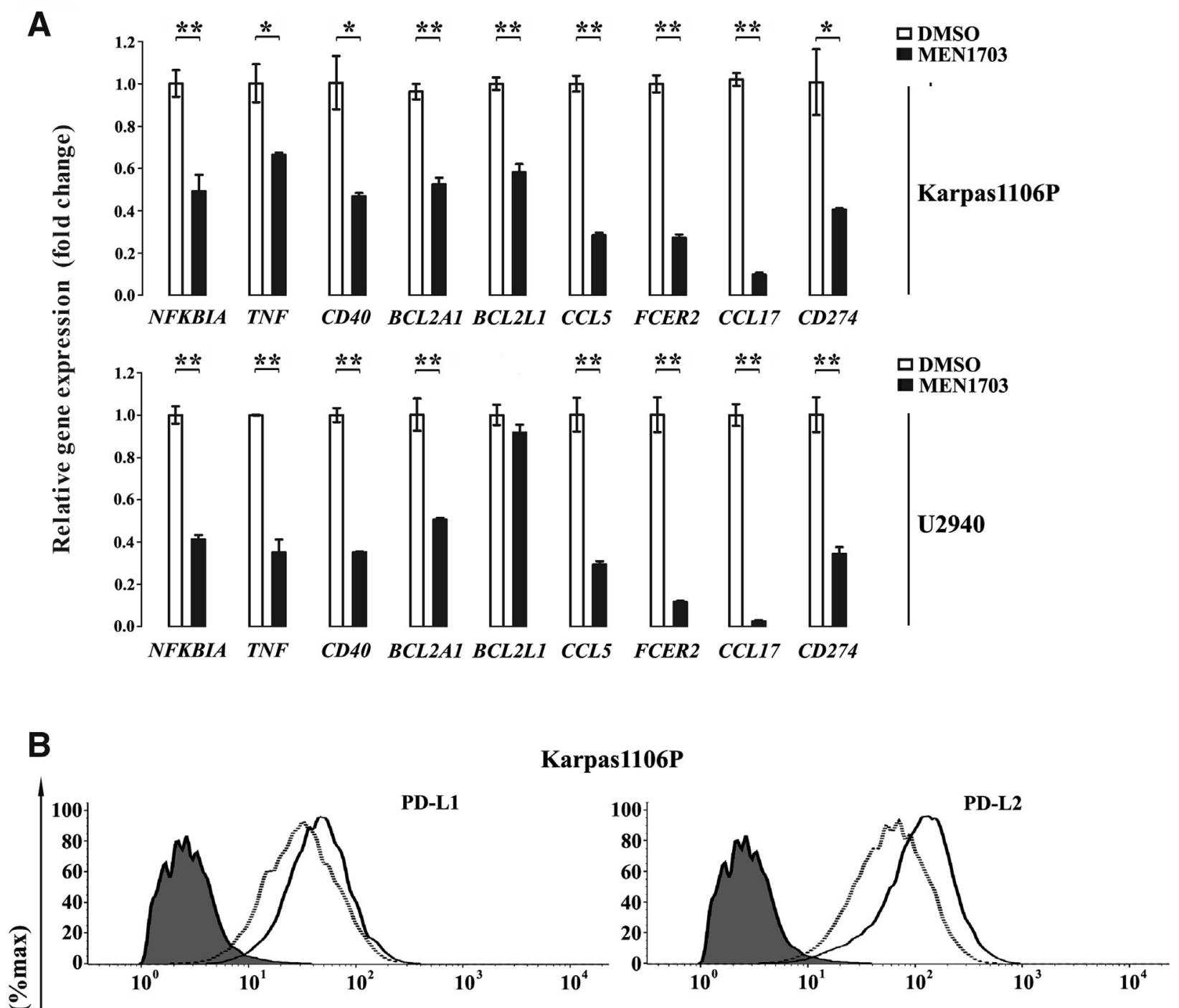

U2940

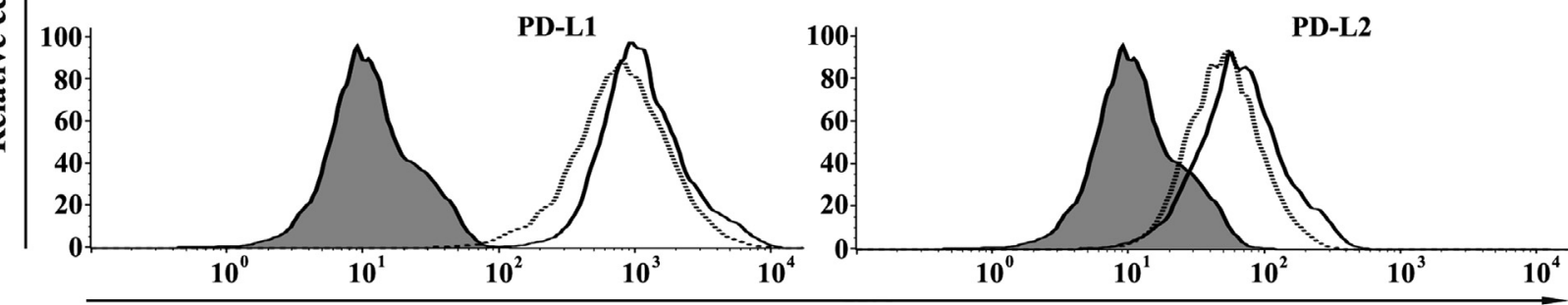

PE

\section{Isotype control \\ DMSO \\ IIIIII MEN1703}

Figure 3 Proviral integration site for Moloney murine leukemia virus (PIM) inhibition down-regulates expression of NF- $\mathrm{KB}$ and STAT targets and programmed

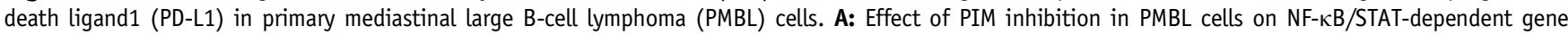
expression. PMBL cells were incubated with dimethyl sulfoxide (DMSO) alone or MEN1703 $(1 \mu \mathrm{mol} / \mathrm{L})$ for 24 hours. Thereafter, gene expression was assessed by real-time quantitative PCR. B: Effect of PIM inhibition on PD-L1 and PD-L2 membrane expression. PMBL cells were incubated with DMSO alone or with MEN1703 (3 $\mu \mathrm{mol} / \mathrm{L})$ for 48 hours. Thereafter, cell surface PD-L1 and PD-L2 expression was assessed by flow cytometry. $\mathrm{n}=3$ independent experiments $(\mathbf{A}$ and $\mathbf{B}) .{ }^{*} P<0.01$, $* * P<0.001$. 
decreased STAT3 (Tyr705) and PIM-specific NF-кB-RelA (S276) phosphorylations (Figure 2D and Supplemental Figure S1C).

\section{PIM Inhibition in PMBL Cells Impairs Prosurvival Factors and Immunosuppressive PD-L1 and PD-L2 Signaling}

In cHL RS cells, PIMs modulate NF- $\mathrm{BB}-$ and STATdependent expression of prosurvival factors, cytokines/chemokines, and immunomodulatory proteins. ${ }^{5}$ To elucidate the role of PIMs in regulation of these molecules in PMBL cells, we investigated transcript levels of direct NF- $\kappa \mathrm{B} / \mathrm{STAT}$ targets (NFKBIA, TNF, CD40, BCL2A1, BCL2L1, CCL5, FCER2, $C C L 17$, and CD274) in Karpas1106P and U2940 cells treated with PIM inhibitors. In line with decreased activity of STAT3 and NF-кB-RelA, MEN1703 and PIM447 significantly down-regulated mRNA levels in most of the investigated genes (Figure 3A and Supplemental Figure S2A). At the protein level, treatment with MEN1703 decreased surface abundance of PD-L1 by $32.89 \%$ and PD-L2 by $48.4 \%$ in Karpas1106P cells and by 26.15\% (PD-L1) and 26.88\% (PD-L2) in U2940 cells (Figure 3B). These observations were confirmed in PMBL cell lines using PIM477 (Supplemental Figure S2B). Collectively, our data highlight the role of PIM kinases in modulating expression of molecules involved in cell survival, communication with tumor microenvironment, and immune escape of PMBL cells.

\section{Discussion}

PMBL and nodular sclerosis -cHL represent two distinct B-cell lymphoma entities with different treatment strategies but similar molecular features, including constitutive NF- $\mathrm{BB}$ and JAKSTAT signaling and expression of immunomodulatory proteins. Our data identify expression of PIM kinases as an additional common feature shared by these two malignant tumors. Similar to cHL, PIM expression in PMBLs exhibits a prosurvival role, modulates protein translation, and augments NF$\kappa \mathrm{B}$ and STAT signaling, facilitating NF- $\kappa \mathrm{B} / \mathrm{STAT}-$ driven expression of cytokines, chemokines, and antiapoptotic and immunomodulatory molecules.

We have previously reported that NF- $\kappa$ B and STAT transcription factors are important inducers of PIM kinase expression in RS cells. ${ }^{5}$ Consistent with the molecular similarity between PMBL and RS cells, PIMs are ubiquitously expressed in PMBL biopsy specimens and PMBL-derived cell lines. Aberrant NF- $\kappa B$ activity in PMBL results from multiple genetic lesions, including REL, BCL10, and MALT1 amplifications, ${ }^{14,15}$ or loss-of-function mutations in $T N F A I P 3^{16}$ and increases the expression of antiapoptotic genes, such as $B C L 2 L 1$ (BCLXL) and BCL2A1 (BFL1). ${ }^{17,18}$ Similarly, activation of JAK kinases and their downstream STAT transcription factors in PMBL is caused by gain-of-function mutations of STAT6 and $I L 4 R,{ }^{19,20}$ amplification of 9p24.1 region harboring JAK2 locus, ${ }^{4}$ or inactivating SOCS1 mutations. $^{21,22}$ As a consequence, deregulated JAK-STAT signaling leads to the up-regulation of factors associated with PMBL cell survival, including BCLXL, CD23 (FCER2), 19,20 and molecules involved in immune evasion, such as chemokine (C-C motif) ligand (CCL) 17, PD-L1, and PD-L2. ${ }^{4,20}$ Despite the central role of NF- $\kappa B$ and JAK-STAT signaling in the pathogenesis of PMBL, ${ }^{17,23}$ agents targeting these pathways have been the subject of only a few pilot clinical studies thus far. In a phase 1 study, combination of ibrutinib (a BTK inhibitor targeting NF- $\kappa$ B activity) with R-ICE (rituximab plus ifosfamide, carboplatin, and etoposide) immunochemotherapy was associated with a substantial response rate in patients with relapsing/remitting primary mediastinal large B-cell lymphoma. ${ }^{24}$ In contrast, all patients treated with JAK2 inhibitor ruxolitinib progressed rapidly after the first or second cycle of treatment. ${ }^{25}$ Given the partial overlap between genes controlled by NFKB and STATs (eg, BCL2L1 and CCL17), simultaneous targeting of both pathways appears to be a promising strategy that could more effectively block prosurvival signaling in PMBL. Inhibition of PIM kinases may represent such a strategy because PIMs directly enhance NF$\kappa \mathrm{B}$ and STAT transcriptional activity through phosphorylation of the NF- $\kappa$ B subunit RelA and STAT3 ${ }^{5,13}$ In line with these pathogenetic dependencies, PIM kinase inhibition with the dual pan-PIM/FLT3 inhibitor MEN1703 or the selective panPIM inhibitor PIM447 decreased NF- $\kappa$ B-and STAT-driven expression of prosurvival molecules, including BCL2Al, $B C L 2 L 1$, and FCER2. Constitutive activity of the JAK-STAT pathway in PMBL cells also leads to the overexpression of immunomodulatory molecules, including CCL17 and PD-1 ligands, which affect composition and function of the tumor microenvironment. Because PIM blockade markedly downregulated their expression, pharmacologic PIM targeting might be associated with increased antitumor immune response in the clinical setting.

Collectively, we show that the expression and function of PIM kinases extend the list of biological similarities between PMBL and cHL. Given the broad spectrum of factors regulated in PMBL cells by PIM kinases, their inhibition might produce pleiotropic effects that include decreased tumor cell survival and enhanced antitumor immune response.

\section{Acknowledgment}

MEN1703 used in the study was kindly provided by Menarini Ricerche.

\section{Author Contributions}

M.Szy., P.J., and A.S.-C. conceived the study and wrote the manuscript; M.Szo., M.P.S., and A.S.-C. obtained and analyzed the clinical samples; A.M.T. revised the manuscript; M.Szy. and S.D. performed experiments. 


\section{Supplemental Data}

Supplemental material for this article can be found at http://doi.org/10.1016/j.ajpath.2020.12.001.

\section{References}

1. Savage KJ: Rare B-cell lymphomas: primary mediastinal, intravascular, and primary effusion lymphoma. Cancer Treat Res 2008, 142:243-264

2. Savage KJ, Monti S, Kutok JL, Cattoretti G, Neuberg D, De Leval L, Kurtin P, Dal Cin P, Ladd C, Feuerhake F, Aguiar RC, Li S, Salles G, Berger F, Jing W, Pinkus GS, Habermann T, Dalla-Favera R, Harris NL, Aster JC, Golub TR, Shipp MA: The molecular signature of mediastinal large B-cell lymphoma differs from that of other diffuse large B-cell lymphomas and shares features with classical Hodgkin lymphoma. Blood 2003, 102:3871-3879

3. Rosenwald A, Wright G, Leroy K, Yu X, Gaulard P, Gascoyne RD, et al: Molecular diagnosis of primary mediastinal B cell lymphoma identifies a clinically favorable subgroup of diffuse large B cell lymphoma related to Hodgkin lymphoma. J Exp Med 2003, 198:851-862

4. Green MR, Monti S, Rodig SJ, Juszczynski P, Currie T, O’Donnell E, Chapuy B, Takeyama K, Neuberg D, Golub TR, Kutok JL, Shipp MA: Integrative analysis reveals selective 9p24.1 amplification, increased PD-1 ligand expression, and further induction via JAK2 in nodular sclerosing Hodgkin lymphoma and primary mediastinal large B-cell lymphoma. Blood 2010, 116:3268-3277

5. Szydlowski M, Prochorec-Sobieszek M, Szumera-Cieckiewicz A, Derezinska E, Hoser G, Wasilewska D, Szymanska-Giemza O, Jablonska E, Bialopiotrowicz E, Sewastianik T, Polak A, Czardybon W, Galezowski M, Windak R, Zaucha JM, Warzocha K, Brzozka K, Juszczynski P: Expression of PIM kinases in ReedSternberg cells fosters immune privilege and tumor cell survival in Hodgkin lymphoma. Blood 2017, 130:1418-1429

6. Swerdlow SH, Campo E, Harris NL, Jaffe ES, Pileri SA, Stein H, Thiele J: WHO Classification of Tumours of Haematopoietic and Lymphoid Tissues. ed 4 Revised, Vol 2. Lyon, IARC, 2017

7. Brault L, Menter T, Obermann EC, Knapp S, Thommen S, Schwaller J, Tzankov A: PIM kinases are progression markers and emerging therapeutic targets in diffuse large B-cell lymphoma. Br J Cancer 2012, 107:491-500

8. Yang Q, Chen LS, Neelapu SS, Miranda RN, Medeiros LJ, Gandhi V: Transcription and translation are primary targets of Pim kinase inhibitor SGI-1776 in mantle cell lymphoma. Blood 2012, 120:3491-3500

9. Jablonska E, Szydłowski M, Białopiotrowicz E, Kiliszek P, Sewastianik T, Polak A, Warzocha K, Czardybon W, Galezowski M, Windak R, Golas A, Brzózka KD, Juszczynski P: A novel pan-PIM kinase inhibitor, SEL24B489, induces apoptosis and inhibits proliferation of diffuse large B-cell lymphoma cells through inhibition of protein translation and attenuation of Myc and NFkB activity. American Society of Hematology Annual Meeting, Vol 126. Orlando, CA, Blood, 2015. pp. 706

10. Bialopiotrowicz E, Gorniak P, Noyszewska-Kania M, Pula B, MakuchLasica H, Nowak G, Bluszcz A, Szydlowski M, Jablonska E, Piechna K, Sewastianik T, Polak A, Lech-Maranda E, Budziszewska BK, WasyleckaJuszczynska M, Borg K, Warzocha K, Czardybon W, Galezowski M, Windak R, Brzozka K, Juszczynski P: Microenvironment-induced PIM kinases promote CXCR4-triggered mTOR pathway required for chronic lymphocytic leukaemia cell migration. J Cell Mol Med 2018, 22: 3548-3559

11. Paino T, Garcia-Gomez A, Gonzalez-Mendez L, San-Segundo L, Hernandez-Garcia S, Lopez-Iglesias AA, Algarin EM, MartinSanchez M, Corbacho D, Ortiz-de-Solorzano C, Corchete LA, Gutierrez NC, Maetos MV, Garayoa M, Ocio EM: The novel pan-PIM kinase inhibitor, PIM447, displays dual antimyeloma and boneprotective effects, and potently synergizes with current standards of care. Clin Cancer Res 2017, 23:225-238
12. Lu J, Zavorotinskaya T, Dai Y, Niu XH, Castillo J, Sim J, Yu J, Wang Y, Langowski JL, Holash J, Shannon K, Garcia PD: Pim2 is required for maintaining multiple myeloma cell growth through modulating TSC2 phosphorylation. Blood 2013, 122:1610-1620

13. Chen LS, Redkar S, Taverna P, Cortes JE, Gandhi V: Mechanisms of cytotoxicity to Pim kinase inhibitor, SGI-1776, in acute myeloid leukemia. Blood 2011, 118:693-702

14. Weniger MA, Gesk S, Ehrlich S, Martin-Subero JI, Dyer MJ, Siebert R, Moller P, Barth TF: Gains of REL in primary mediastinal Bcell lymphoma coincide with nuclear accumulation of REL protein. Genes Chromosomes Cancer 2007, 46:406-415

15. Wessendorf S, Barth TF, Viardot A, Mueller A, Kestler HA, Kohlhammer H, Lichter P, Bentz M, Dohner H, Moller P, Schwaenen C: Further delineation of chromosomal consensus regions in primary mediastinal B-cell lymphomas: an analysis of 37 tumor samples using high-resolution genomic profiling (array-CGH). Leukemia 2007, 21:2463-2469

16. Schmitz R, Hansmann ML, Bohle V, Martin-Subero JI, Hartmann S, Mechtersheimer G, Klapper W, Vater I, Giefing M, Gesk S, Stanelle J, Siebert R, Kuppers R: TNFAIP3 (A20) is a tumor suppressor gene in Hodgkin lymphoma and primary mediastinal B cell lymphoma. J Exp Med 2009, 206:981-989

17. Lam LT, Davis RE, Pierce J, Hepperle M, Xu Y, Hottelet M, Nong Y, Wen D, Adams J, Dang L, Staudt LM: Small molecule inhibitors of IkappaB kinase are selectively toxic for subgroups of diffuse large Bcell lymphoma defined by gene expression profiling. Clin Cancer Res $2005,11: 28-40$

18. Hinz M, Loser P, Mathas S, Krappmann D, Dorken B, Scheidereit C: Constitutive NF-kappaB maintains high expression of a characteristic gene network, including CD40, CD86, and a set of antiapoptotic genes in Hodgkin/Reed-Sternberg cells. Blood 2001, 97:2798-2807

19. Ritz O, Guiter C, Castellano F, Dorsch K, Melzner J, Jais JP, Dubois G, Gaulard P, Moller P, Leroy K: Recurrent mutations of the STAT6 DNA binding domain in primary mediastinal B-cell lymphoma. Blood 2009, 114:1236-1242

20. Vigano E, Gunawardana J, Mottok A, Van Tol T, Mak K, Chan FC, Chong L, Chavez E, Woolcock B, Takata K, Twa D, Shulha HP, Telenius A, Kutovaya O, Hung SS, Healy S, Ben-Neriah S, Leroy K, Gaulard P, Diepstra A, Kridel R, Savage KJ, Rimsza L, Gascoyne R, Steidl C: Somatic IL4R mutations in primary mediastinal large B-cell lymphoma lead to constitutive JAK-STAT signaling activation. Blood 2018, 131:2036-2046

21. Melzner I, Bucur AJ, Bruderlein S, Dorsch K, Hasel C, Barth TF, Leithauser F, Moller P: Biallelic mutation of SOCS-1 impairs JAK2 degradation and sustains phospho-JAK2 action in the MedB-1 mediastinal lymphoma line. Blood 2005, 105:2535-2542

22. Melzner I, Weniger MA, Bucur AJ, Bruderlein S, Dorsch K, Hasel C, Leithauser F, Ritz O, Dyer MJ, Barth TF, Moller P: Biallelic deletion within 16p13.13 including SOCS-1 in Karpas1106P mediastinal B-cell lymphoma line is associated with delayed degradation of JAK2 protein. Int J Cancer 2006, 118:1941-1944

23. Hao Y, Chapuy B, Monti S, Sun HH, Rodig SJ, Shipp MA: Selective JAK2 inhibition specifically decreases Hodgkin lymphoma and mediastinal large B-cell lymphoma growth in vitro and in vivo. Clin Cancer Res 2014, 20:2674-2683

24. Sauter CS, Matasar MJ, Schoder H, Devlin SM, Drullinsky P, Gerecitano J, Kumar A, Noy A, Palomba ML, Portlock CS, Straus DJ, Zelenetz AD, McCall SJ, Miller ST, Courtien AI, Younes A, Moskowitz CH: A phase 1 study of ibrutinib in combination with RICE in patients with relapsed or primary refractory DLBCL. Blood 2018, 131:1805-1808

25. Kim SJ, Kang HJ, Dong-Yeop S, Lee HS, Yong S, Shin H, Yoon DH, Hong JY, Kong JH, Sakamoto K, Ko YH, Takeuchi K: The efficacy of JAK2 inhibitor in heavily pretreated classical hodgkin lymphoma: a prospective pilot study of ruxolitinib in relapsed or refractory classical Hodgkin lymphoma and primary mediastinal large B-cell lymphoma. Blood 2016, 128:1820 